Supporting Information for:

\title{
Spin-Controlled Charge Recombination Pathways across the Inorganic/Organic Interface
}

Junhui Wang, ${ }^{1}$ Tao Ding, ${ }^{1}$ Chengming Nie, ${ }^{2}$ Mei Wang, ${ }^{2}$ Panwang Zhou ${ }^{3}$ and Kaifeng $W u^{1 *}$

${ }^{1}$ State Key Laboratory of Molecular Reaction Dynamics and Dynamics Research Center for Energy and Environmental Materials, Dalian Institute of Chemical Physics, Chinese Academy of Sciences, Dalian, Liaoning 116023, China

2 State Key Laboratory of Fine Chemicals, Institute of Artificial Photosynthesis, Dalian University of Technology, Dalian, Liaoning 116024, China

${ }^{3}$ Institute of Molecular Sciences and Engineering, Institute of Frontier and Interdisciplinary Science, Shandong University, Qingdao, Shandong 266235, China

Address correspondence to: kwu@dicp.ac.cn

\section{Content list:}

Figures S1-S12

Notes 1-3

Tables S1 and S2 


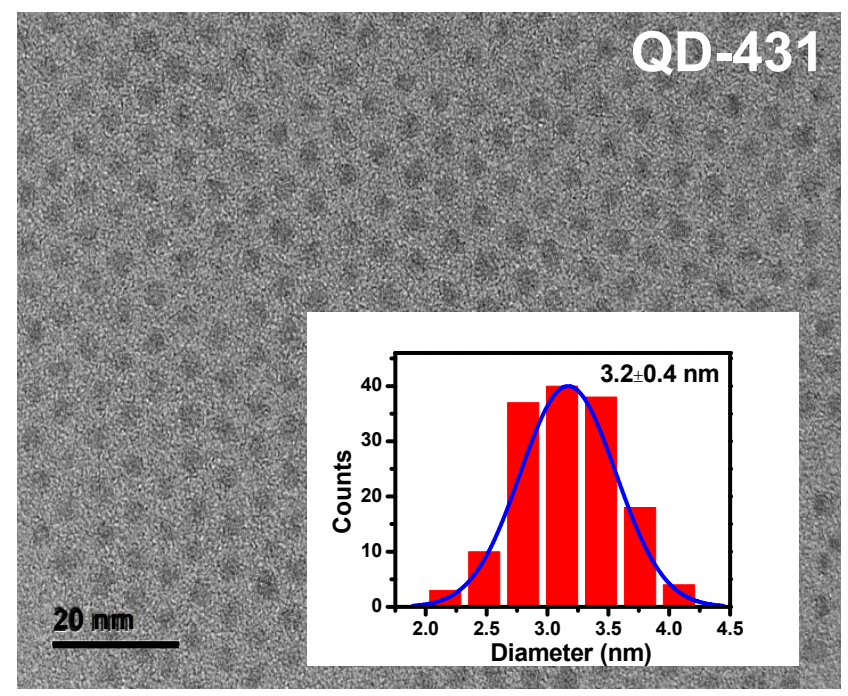

Figure S1. TEM image and size distribution histogram of the CdS QDs with their first excitonic absorption peak at $431 \mathrm{~nm}$ used in this study. 

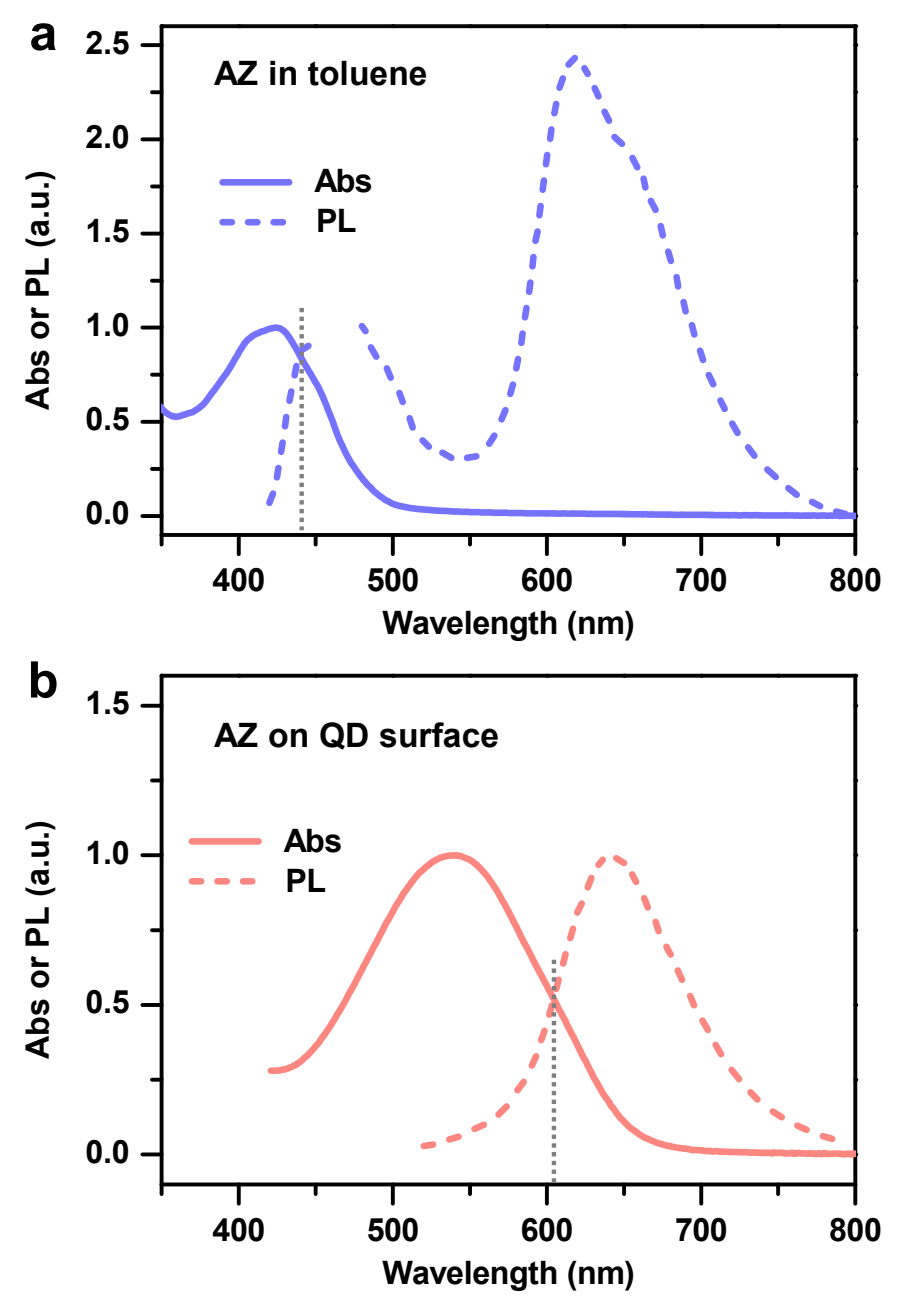

Figure S2. Absorption (solid) and PL (dashed) spectra of (a) free AZs in toluene and (b) AZs adsorbed on CdS QD surfaces. The absorption spectrum of the latter was obtained by performing a subtraction between QD-AZ complexes and QDs. The intersections between the normalized absorption and PL spectra were used to determine the optical gaps of free AZs and AZs on QDs, ${ }^{1}$ which are $440 \mathrm{~nm}(2.82 \mathrm{eV})$ and $605 \mathrm{~nm}(2.05 \mathrm{eV})$, respectively. Note that the break at $\sim 460 \mathrm{~nm}$ for the first PL peak of free AZs in toluene solution corresponds to the Raman bands due to the very low PL QY of AZs $(\sim 0.05 \%) .^{2}$ The PL spectrum of free AZs also has a second PL peak at $\sim 620 \mathrm{~nm}$ that has been well documented and explained as the emission of the excited state intra-molecular proton transfer (ESIPT) tautomer of AZs. ${ }^{2,3}$ 


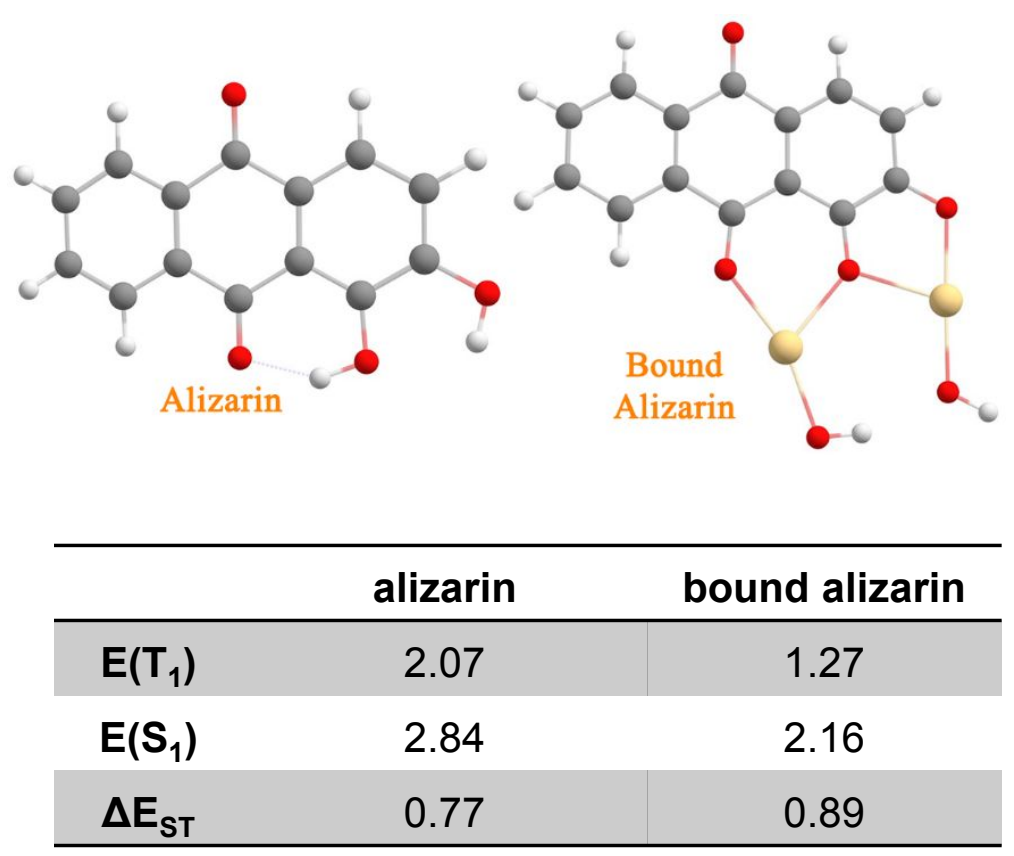

Figure S3. B3LYP-D3/TZVP optimized geometry of free AZs and model compound of CdS-surface-bound AZs (C: gray; H: white; O: red; Cd: yellow). The calculated $S_{1}$ and $T_{1}$ transition energies are 2.84 and $2.07 \mathrm{eV}$, respectively, for free AZs and are 2.16 and $1.27 \mathrm{eV}$, respectively, for AZs on QDs. The singlet-triplet splitting $\left(\Delta E_{\mathrm{ST}}\right)$ values are 0.77 and $0.89 \mathrm{eV}$, respectively, for free $\mathrm{AZs}$ and $\mathrm{AZs}$ on $\mathrm{QDs}$, consistent with the strong exchange interaction expected for small organic molecules. 

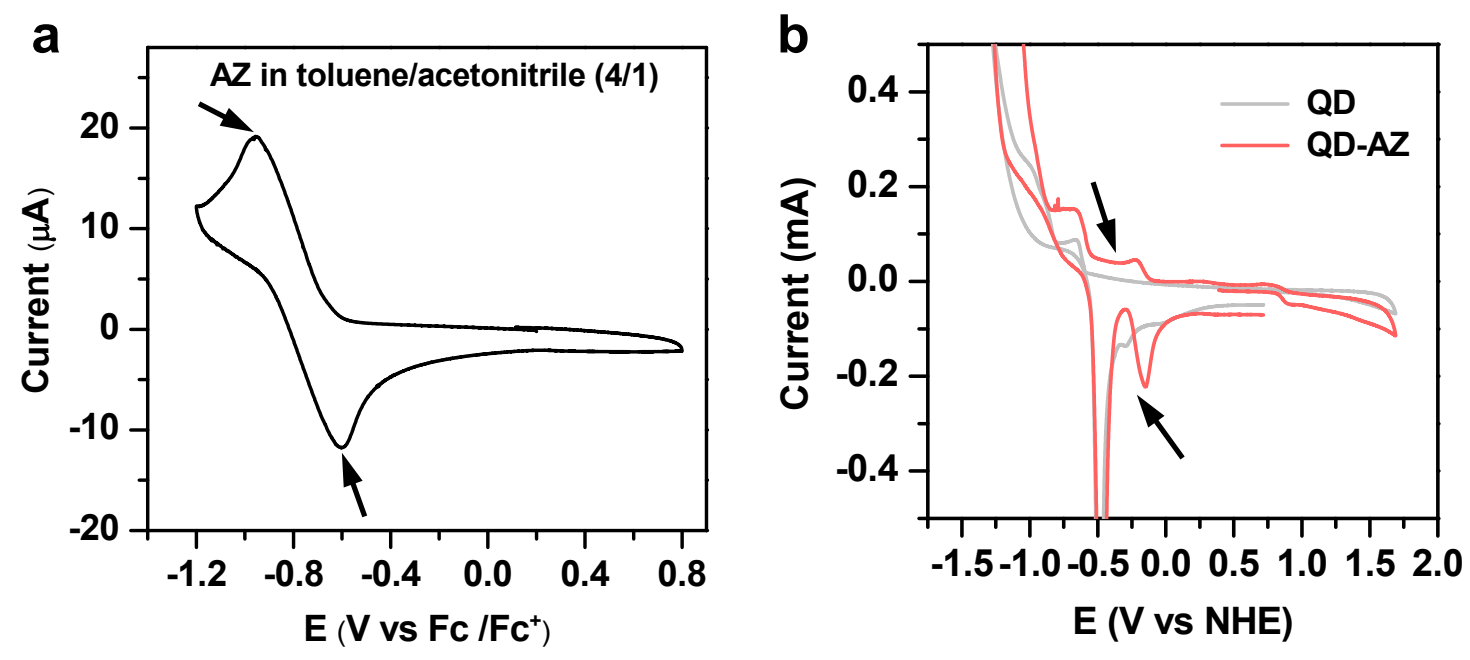

Figure S4. (a) Cyclic voltammogram (CV) of free AZs dissolved in a mixture of toluene and acetonitrile $(\mathrm{v} / \mathrm{v}=4 / 1)$ containing tetra-butyl ammonium perchlorate $(0.1 \mathrm{M})$ as the electrolyte, with a scan rate of $100 \mathrm{mV} / \mathrm{s}$. The measurement was calibrated using the Ferrocene/Ferrocenium couple $\left(\mathrm{Fc} / \mathrm{Fc}^{+}\right)$. The indicated redox peaks at $-0.60 \mathrm{~V}$ and $-0.96 \mathrm{~V}$ (vs. $\left.\mathrm{Fc} / \mathrm{Fc}^{+}\right)$can be attributed to the LUMO level of AZs: $E_{\mathrm{HOMO}}=-\left[\left(\mathrm{e} V^{\mathrm{ox}}+\mathrm{e} V^{\mathrm{red}}\right) / 2+4.8 \mathrm{eV}\right]$ $=-4.0 \mathrm{eV}$ ( $v s$. vacuum), where $4.8 \mathrm{eV}$ is the energy of $\mathrm{Fc} / \mathrm{Fc}^{+} v s$. vacuum. (b) $\mathrm{CVs}$ of films of free CdS QDs (gray) and CdS QD-AZ complexes (red) immersed in $0.5 \mathrm{M}$ aqueous $\mathrm{Na}_{2} \mathrm{SO}_{4}$ with a scan rate of $100 \mathrm{mV} / \mathrm{s}$. The measurement was calibrated using the $\mathrm{Ru}(\mathrm{bpy})_{3}{ }^{2+} /$ $\mathrm{Ru}(\mathrm{bpy}) 3^{3+}$ couple. The additional redox peaks at $-0.25 \mathrm{~V}$ and $-0.15 \mathrm{~V}$ (vs. NHE) can be attributed to the HOMO level of $\mathrm{AZ}$ adsorbed on QD surfaces: $E_{\mathrm{HOMO}}=-\left[\left(\mathrm{e} V^{\mathrm{ox}}+\mathrm{e} V^{\mathrm{red}}\right) / 2+\right.$ $4.5 \mathrm{eV}]=-4.3 \mathrm{eV}$ (vs. vacuum), where $4.5 \mathrm{eV}$ is the energy of NHE $v s$. vacuum. The LUMO energy level can be estimated using the HOMO energy level and the optical gap of AZ adsorbed on QD surface $(605 \mathrm{~nm}$; or $2.05 \mathrm{eV})$ : $E_{\mathrm{LUMO}}=-4.3 \mathrm{eV}+2.05 \mathrm{eV}=-2.25 \mathrm{eV}(v s$. vacuum). 

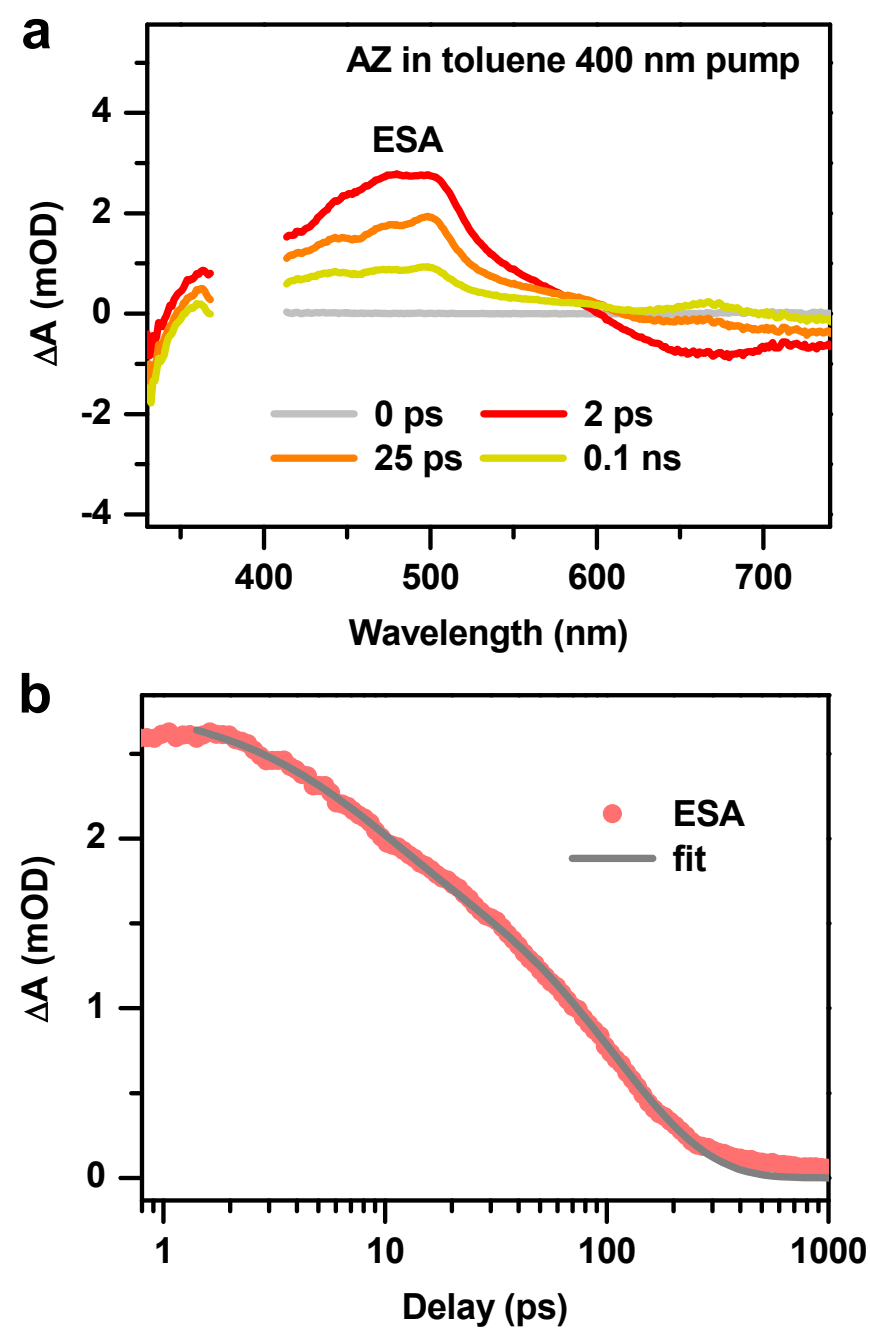

Figure S5. (A) TA spectra of free AZs in toluene at selective delays following $400 \mathrm{~nm}$ excitation. (c) TA kinetics probed at the excited state absorption (ESA) feature $\sim 485 \mathrm{~nm}$ and its fit to a bi-exponential decay with time constants (and relative amplitudes) of 7.5 ps $(29.5 \%)$ and 108 ps (70.5\%). The amplitude-average lifetime is 78.4 ps. 

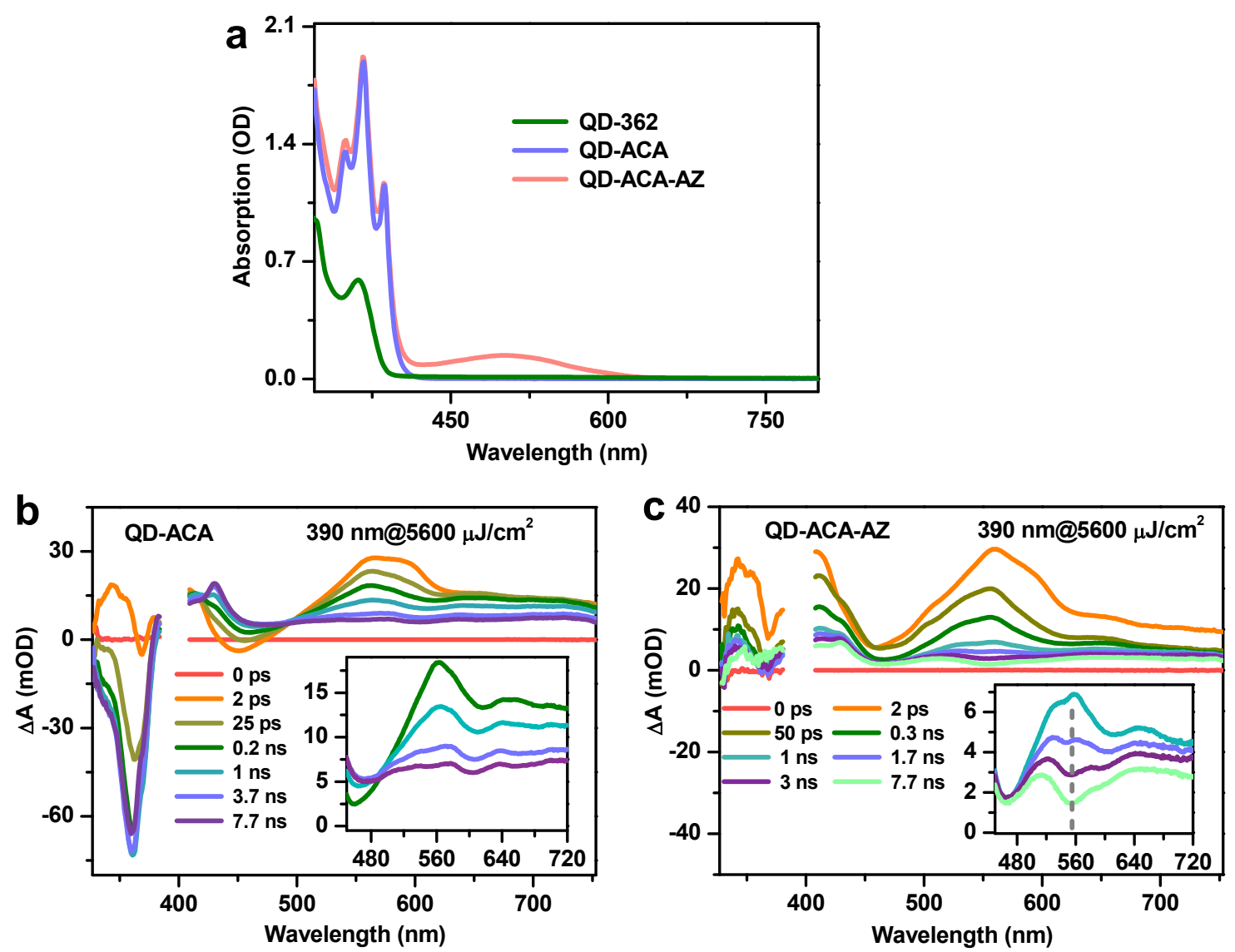

Figure S6. (a) Absorption spectra of free CdS QDs of small sizes (peak at 362 nm; green), QD-ACA (blue) and QD-ACA-AZ complexes (red) dispersed in hexane. ACA is the triplet sensitizer for AZ. (b,c) TA spectra of QD-ACA and QD-ACA-AZ complexes generated by selectively exciting ACA using $390 \mathrm{~nm}$ pump pulses. Insets in (b,c) show the enlarged views of photoinduced absorption (PIA) signals of ACA. An additional bleach feature centered at $560 \mathrm{~nm}$ overlapped with the PIA signal of ACA is detected for the QD-ACA-AZ complexes. By subtracting the background PIA signal of ACA, the TA feature of triplet states of AZ $\left({ }^{3} \mathrm{AZ} *\right)$ can be obtained, which is plotted in Fig. $3 \mathrm{~d}$ in the main text. 

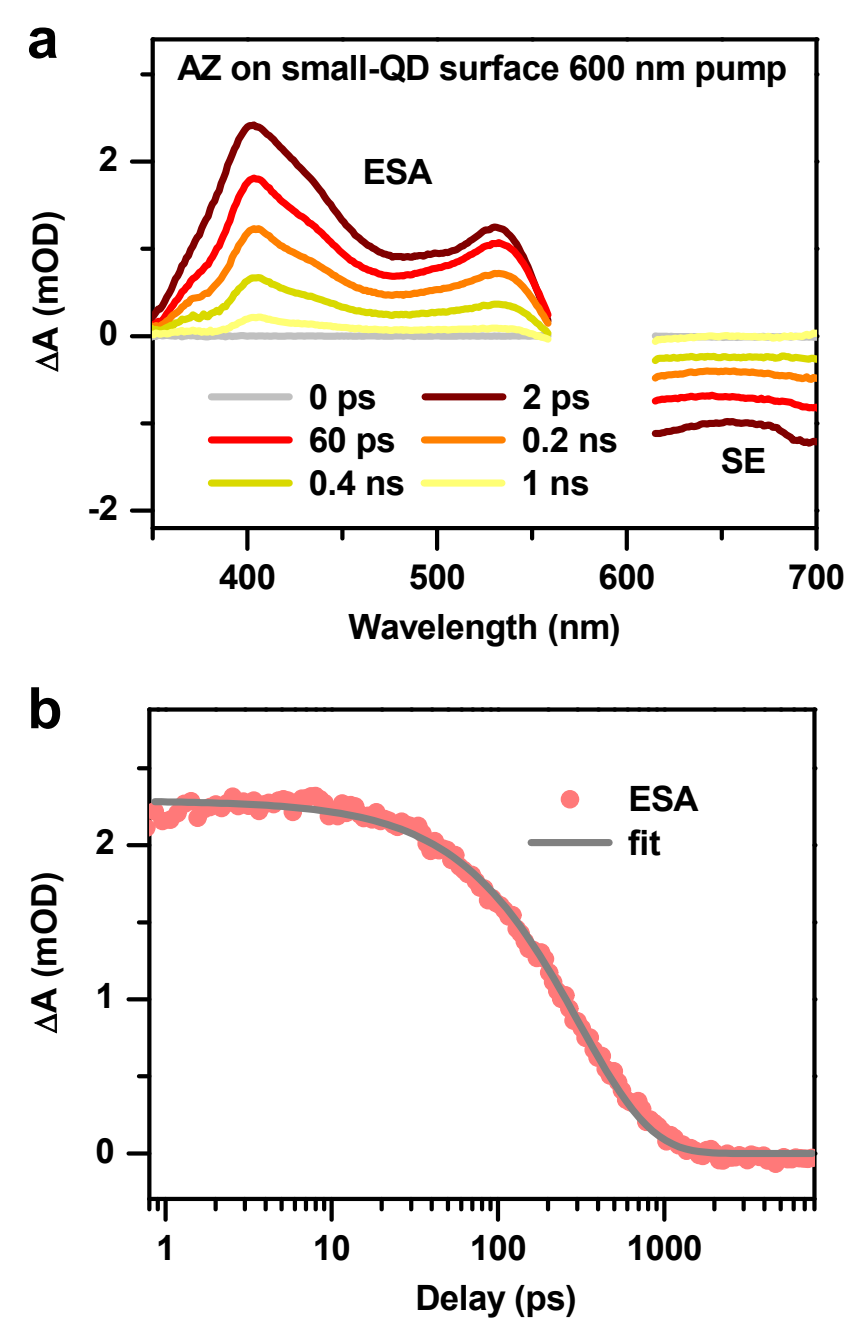

Figure S7. (a) TA spectra of AZs adsorbed on small-size CdS QD surfaces probed at indicated time delays following $600 \mathrm{~nm}$ excitation. In this case, ET from excited AZs to QDs is energetically inhibited. The TA spectra show excited state absorption (ESA) and stimulated emission (SE) of AZs. (b) TA kinetics probed at the ESA (red circles) and its fit to a single-exponential decay with a time constant of $\sim 310 \mathrm{ps.}$ 

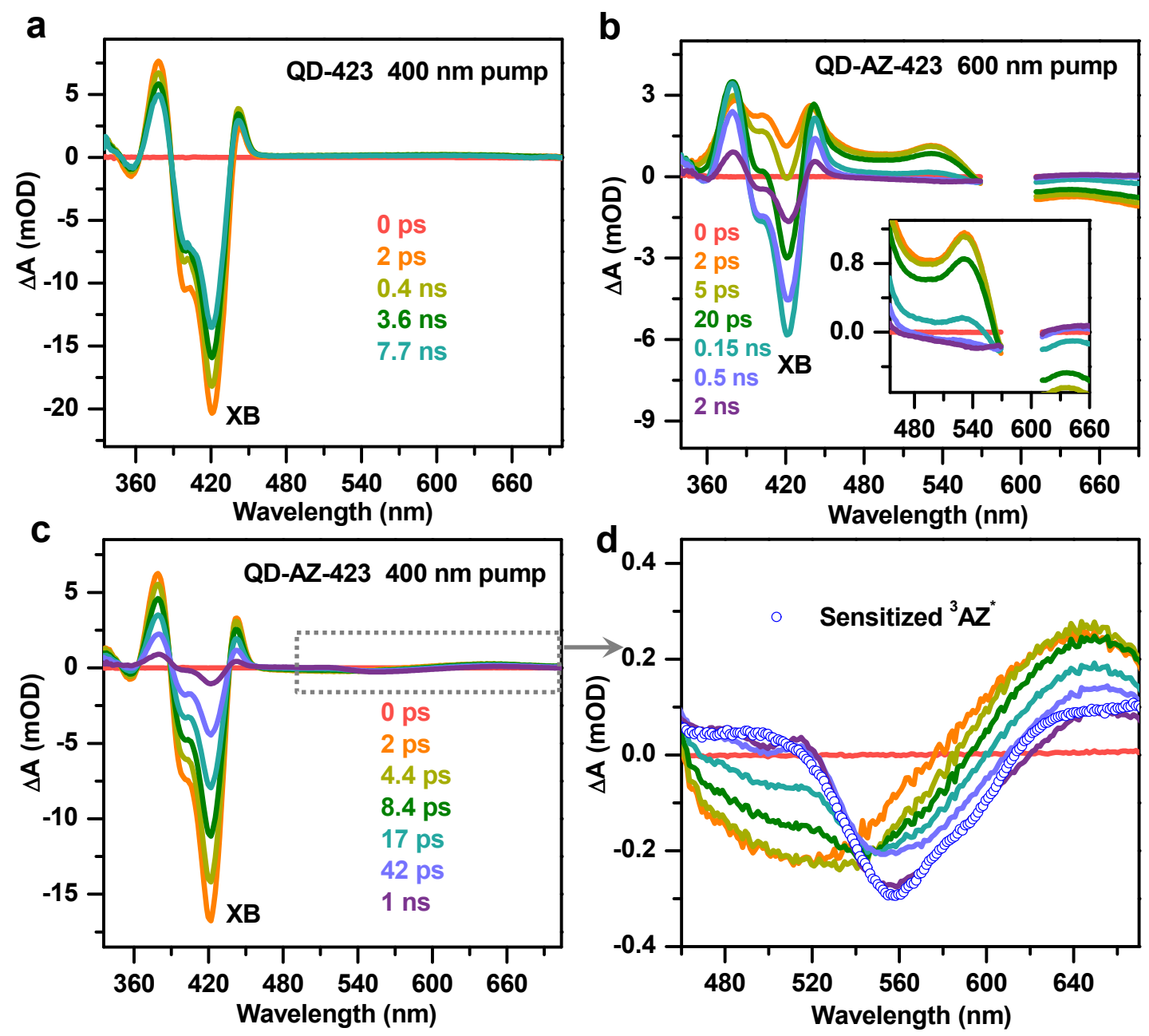

Figure S8. (a) TA spectra of free CdS QD-423 nm following $400 \mathrm{~nm}$ excitation. $(\mathrm{b}, \mathrm{c}) \mathrm{TA}$ spectra of QD-AZ complexes following (b) $600 \mathrm{~nm}$ and (c) $400 \mathrm{~nm}$ excitations. Inset in (b) shows the enlarged view of AZ signals. (d) Enlarged view of AZ signals in (c). The ${ }^{3} \mathrm{AZ} *$ spectrum obtained by triplet sensitization (blue circles) is also shown for comparison. 

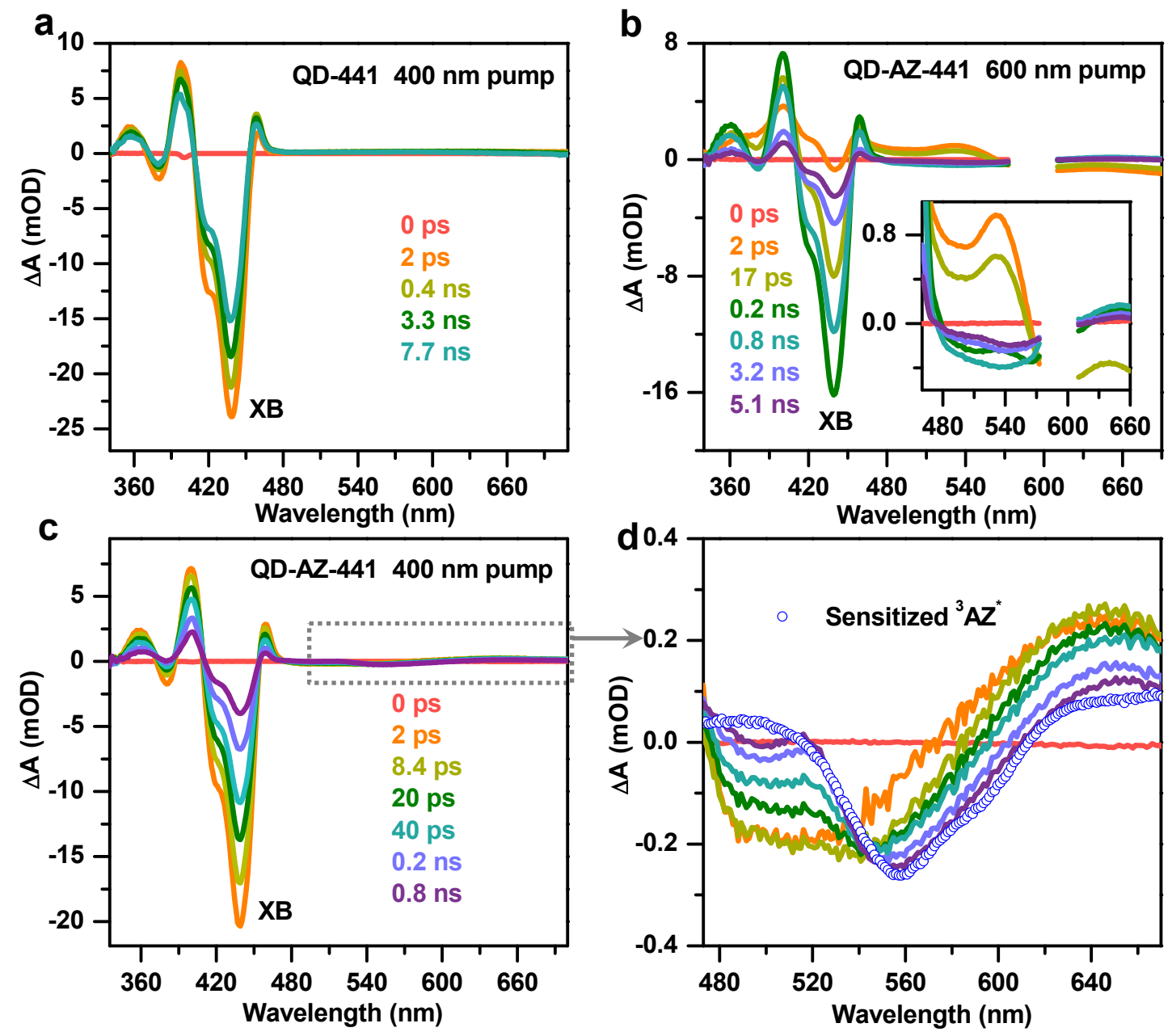

Figure S9. (a) TA spectra of free CdS QD-441 nm following $400 \mathrm{~nm}$ excitation. $(\mathrm{b}, \mathrm{c}) \mathrm{TA}$ spectra of CdS QD-AZ complexes following (b) $600 \mathrm{~nm}$ and (c) $400 \mathrm{~nm}$ excitations. Inset in (b) shows the enlarged view of AZ signals. (d) Enlarged view of AZ signals in (c). The ${ }^{3} \mathrm{AZ} *$ spectrum obtained by triplet sensitization (blue circles) is also shown for comparison. 


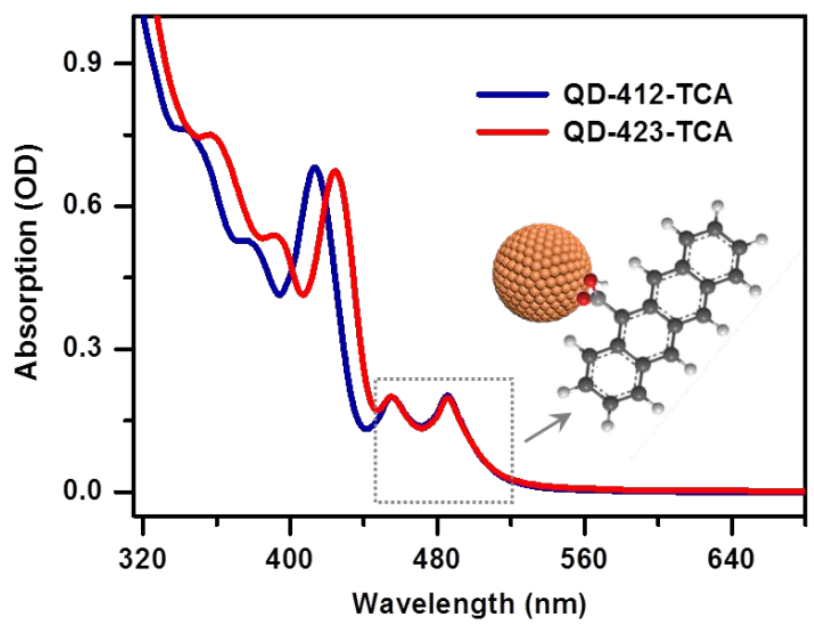

Figure S10. Absorption spectra of CdS QD-TCA complexes. The molecular structure and the absorption features of TCA are indicated. The two QDs have lowest energy absorption peaks at 412 and $423 \mathrm{~nm}$.
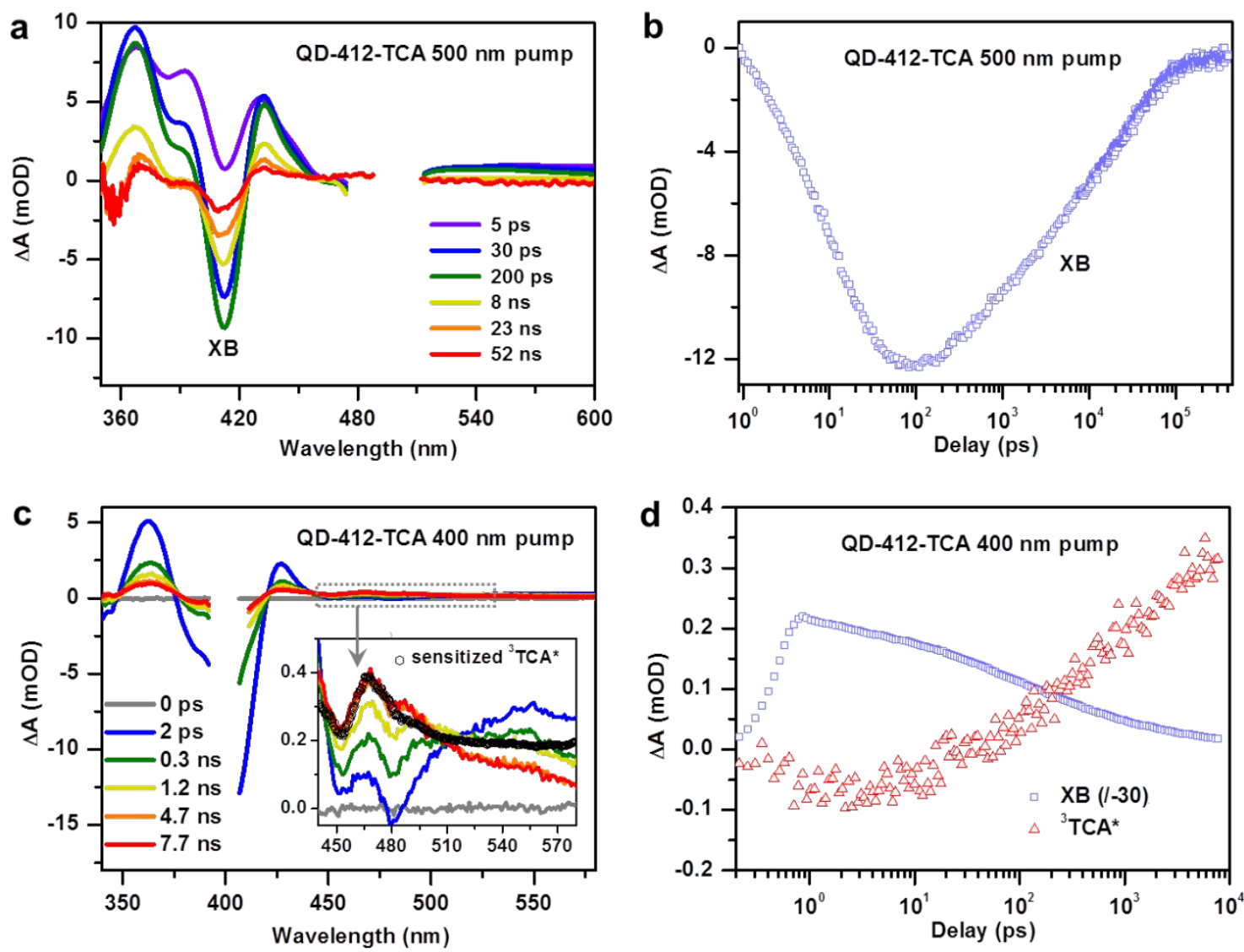

Figure S11. (a) TA spectra of CdS QD-412-TCA complexes at indicated delays following the excitation by $500 \mathrm{~nm}$ pulses selectively pumping TCA. (b) TA kinetics probed at the XB 
feature, showing the formation and decay of $\mathrm{XB}$ due to electron injection from photoexcited TCA to QDs and charge recombination, respectively. No long-lived TCA triplet features are observed at the end, indicating that CR1 is the dominating pathway in this case, similar to the observation for QD-AZ systems. (c) TA spectra at indicated delays following the excitation by $400 \mathrm{~nm}$ pulses selectively pumping QDs. Inset show the zoom-in of the TCA signals, which gradually evolves into a long-lived spectrum resembling that of sensitized TCA triplets. (d) TA kinetics probed at the XB (blue) and TCA triplets (red), showing that the decay of $\mathrm{XB}$ is complemented by the formation of TCA triplets. Note that the $\mathrm{XB}$ feature has been inverted and divided by a factor of 30 for clarity.
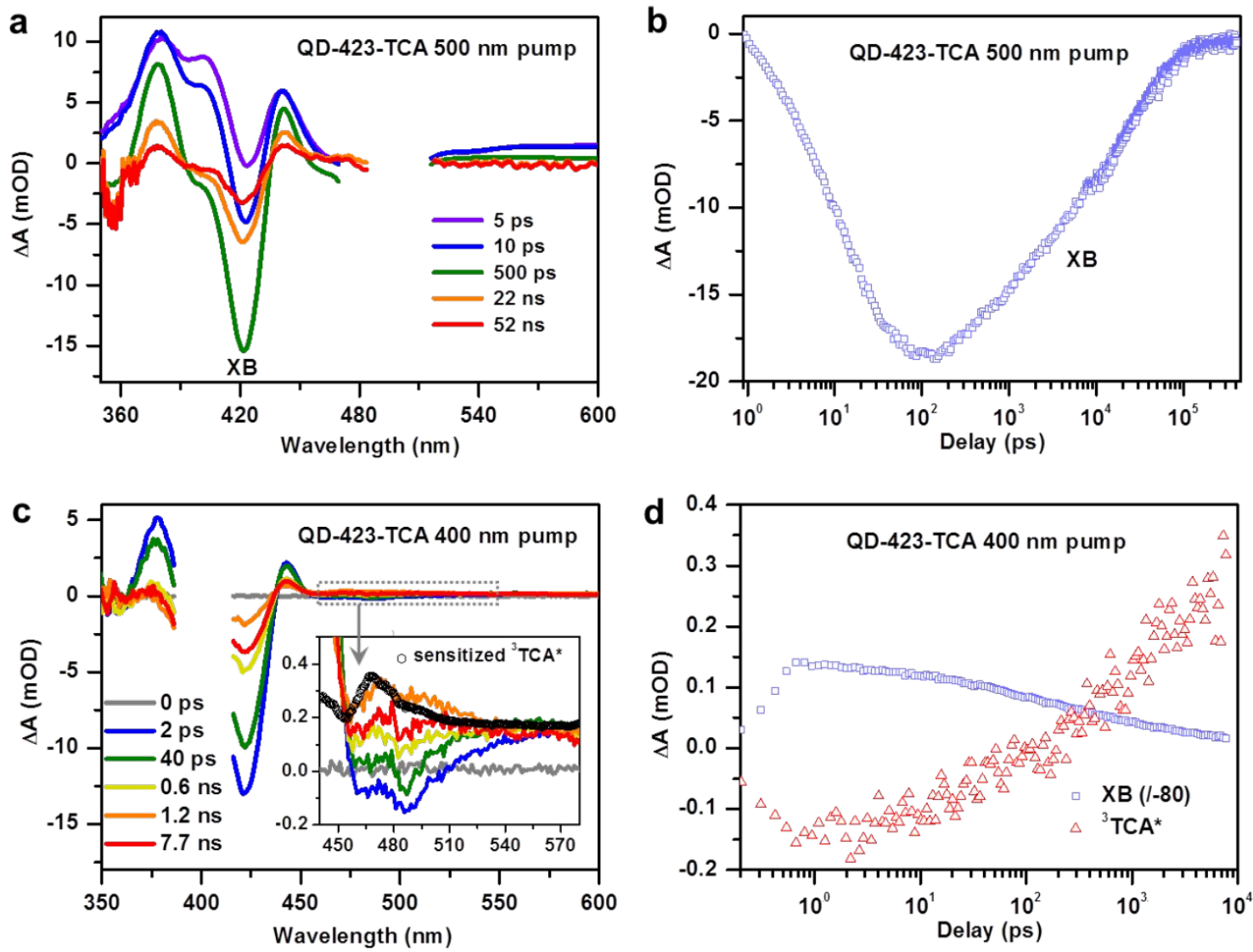
Figure S12. (a) TA spectra of CdS QD-423-TCA complexes at indicated delays following the excitation by $500 \mathrm{~nm}$ pulses selectively pumping TCA. (b) TA kinetics probed at the XB feature, showing the formation and decay of $\mathrm{XB}$ due to electron injection from photoexcited TCA to QDs and charge recombination, respectively. No long-lived TCA triplet features are observed at the end, indicating that $\mathrm{CR} 1$ is the dominating pathway in this case, similar to the observation for QD-AZ systems. (c) TA spectra at indicated delays following the excitation by $400 \mathrm{~nm}$ pulses selectively pumping QDs. Inset show the zoom-in of the TCA signals, which gradually evolves into a long-lived spectrum resembling that of sensitized TCA triplets. (d) TA kinetics probed at the XB (blue) and TCA triplets (red), showing that the decay of $\mathrm{XB}$ is complemented by the formation of TCA triplets. Note that the XB feature has been inverted and divided by a factor of 70 for clarity.

\section{Note 1. Estimation of QD band edges.}

Ingole et al. measured the lowest energy electron $\left(E_{e}\right)$ and hole $\left(E_{h}\right)$ levels of CdS QDs capped with oleic acid ligands using cyclic voltammetry $(\mathrm{CV}) .{ }^{4}$ According to their results, $E_{e}$ and $E_{h}$ for CdS QDs with the $1 S$ absorption peak at $410 \mathrm{~nm}$ are -2.38 and $-5.38 \mathrm{eV}$, respectively, vs. vacuum. Our CdS QDs have the $1 S$ absorption peak at $430 \mathrm{~nm}$, and we can estimate their $E_{e}$ and $E_{h}$ using the reported reference values according the following equations: ${ }^{5}$

$$
E_{e}=E_{e, r e f}+\frac{m_{h}}{m_{e}+m_{h}}\left(E_{g}-E_{g, r e f}\right)
$$




$$
E_{h}=E_{h, r e f}-\frac{m_{e}}{m_{e}+m_{h}}\left(E_{g}-E_{g, r e f}\right)
$$

where $E_{g}$ is the optical band gap, ref denotes the values form the reference QDs, and $m_{e}$ and $m_{h}$ are the electron ( 0.2 free electron mass) and hole ( 0.7 free electron mass $)$ effective masses, respectively. Using eqs. S1 and $\mathrm{S} 2, E_{e}$ and $E_{h}$ of our QDs are calculated to be $\sim-2.5 \mathrm{eV}$ and $-5.35 \mathrm{eV} v s$. vacuum, respectively, as indicated in Fig. 1b. Note that in using eqs. S1 and S2, we have assumed that e-h coulomb binding energies are similar between our QDs and the reference QDs.

\section{Note 2. Kinetics fitting models}

Interfacial charge transfer (CT) between molecules and semiconductors are almost universally multi-exponentials due to heterogeneities in binding numbers and geometries. For QD-AZ samples under $600 \mathrm{~nm}$ excitation, bi-exponentials are required to describe both ET and $\mathrm{CR} 1$ processes. Thus, the kinetics for XB feature of QDs can be expressed as:

$$
S_{X B, Q D}(t) \propto \sum_{i=1}^{2} A_{i}\left(e^{-k_{E T, i} t}-e^{-k_{C R 1, i} t}\right)
$$

where $A_{i}, k_{E T, i}$, and $k_{C R 1, i}$ are the amplitude, ET rate constant, and CR1 rate constant, respectively, of the $i$-th component.

For QD-AZ samples under $400 \mathrm{~nm}$ excitation, the kinetics of the XB feature of QDs was dominated by $\mathrm{CR} 2$ between $\mathrm{QD}^{-}$and $\mathrm{AZ}^{+}$to form the triplet states of $\mathrm{AZ}$, which required fitting using a three-exponential decay function:

$$
S_{X B, Q D}(t) \propto \sum_{i=1}^{3} A_{i} e^{-k_{C R 2, i} t}
$$

where $A_{i}, k_{C R 2, i}$ are the amplitude and CR2 rate constant of the $i$-th component, respectively. 
The fitting parameters are tabulated in Supplementary Tables 1 and 2, respectively; note that the rate constants in the above equations are all converted to time constants in the tables.

Table S1. Time constants for QD-AZ complexes under $600 \mathrm{~nm}$ excitation.

\begin{tabular}{|c|c|c|c|c|c|c|}
\hline $600 \mathrm{~nm}$ & $\tau_{E T, 1}(\mathrm{ps})$ & $\tau_{E T, 2}(\mathrm{ps})$ & $\tau_{E T, a v e}(\mathrm{ps})$ & $\tau_{C R 1,1}(\mathrm{ps})$ & $\tau_{C R 1,2}(\mathrm{ps})$ & $\tau_{C R 1, \text { ave }}(\mathrm{ps})$ \\
pump & $\left(A_{1}\right)$ & $\left(A_{2}\right)$ & & $\left(A_{1}\right)$ & $\left(A_{2}\right)$ & \\
\hline QD-AZ & $9.26 \pm 0.34$ & $58.8 \pm 2.77$ & $40.3 \pm 1.9$ & $769.2 \pm 23.7$ & $3333.3 \pm 111.1$ & $1728.2 \pm 56.4$ \\
& $37.4 \%$ & $62.6 \%$ & & $62.6 \%$ & $37.4 \%$ & \\
\hline
\end{tabular}

Table S2. Time constants for QD-AZ complexes under $400 \mathrm{~nm}$ excitation.

\begin{tabular}{|c|c|c|c|c|c|}
\hline \multirow{2}{*}{$400 \mathrm{~nm}$} & \multicolumn{4}{|c|}{$\tau_{C R 2}(\mathrm{ps})$} & $\tau_{H T}(\mathrm{ps})$ \\
\hline pump & $\tau_{C R 2,1}(\mathrm{ps})$ & $\tau_{C R 2,2}(\mathrm{ps})$ & $\tau_{C R 2,3}(\mathrm{ps})$ & $\tau_{C R 2, \text { ave }}(\mathrm{ps})$ & \\
\hline QD-AZ & $10.7 \pm 0.34$ & $97.1 \pm 4.71$ & $2380.9 \pm 113$. & $444.1 \pm 21.1$ & $0.67 \pm 0.07$ \\
& $55.7 \%$ & $27.0 \%$ & 4 & & \\
& & & $\left(A_{2}\right)$ & & \\
& & & $17.3 \%$ & & \\
\hline
\end{tabular}

Note 3. Estimation of ${ }^{3} \mathrm{AZ} *$ yield for $\mathrm{QD}-\mathrm{AZ}$ complexes under $600 \mathrm{~nm}$ excitation

The yield of ${ }^{3} \mathrm{AZ} *$ formation $\left(\Phi\left({ }^{3} \mathrm{AZ} *\right)\right)$ under $600 \mathrm{~nm}$ excitation was estimated using the signal amplitudes of the kinetic curve probed at $\sim 565 \mathrm{~nm}$ (Fig. 3a). At $\sim 200 \mathrm{ps,} \mathrm{the} \mathrm{signal}$ reaches its maximum amplitude of $\sim-0.35 \mathrm{mOD}$, which can be assigned to the $\mathrm{AZ}^{+}$formed by ET from $\mathrm{AZ} *$ to $\mathrm{QD}\left(S\left(\mathrm{AZ}^{+}\right)\right)$. At $\sim 8 \mathrm{~ns}$, the remaining signal amplitude at $565 \mathrm{~nm}$ is $\sim-0.1$ 
mOD, which can be attributed to the contribution from ${ }^{3} \mathrm{AZ} *\left(S\left({ }^{3} \mathrm{AZ} *\right)\right)$. This is because $\mathrm{AZ}^{+}$ is already annihilated via CR1, as indicated by the near complete recovery of the XB of QDs in $8 \mathrm{~ns}$.

The relative extinction coefficient of the TA spectra of ${ }^{3} \mathrm{AZ}^{*}$ and $\mathrm{AZ}^{+}$at $565 \mathrm{~nm}$, $\varepsilon\left({ }^{3} \mathrm{AZ} Z^{*}\right) / \varepsilon\left(\mathrm{AZ}^{+}\right)$, can be derived from the TA spectra in Fig. $3 \mathrm{~d}$. The spectra at 2 ps and $3 \mathrm{~ns}$ can be assigned to pure $\mathrm{AZ}^{+}$and ${ }^{3} \mathrm{AZ} *$, respectively, on the basis of the kinetics of $\mathrm{HT}$ and $\mathrm{CR} 2$ in the $400 \mathrm{~nm}$ excited QD-AZ complexes. Thus, $\varepsilon\left({ }^{3} \mathrm{AZ} *\right) / \varepsilon\left(\mathrm{AZ}^{+}\right)$is $\sim 0.27 / 0.054=5$.

$\Phi\left({ }^{3} \mathrm{AZ}\right)$ can be calculated using:

$$
\Phi\left({ }^{3} \mathrm{AZ} *\right)=\left[\frac{S\left({ }^{3} \mathrm{AZ}{ }^{*}\right)}{S\left(\mathrm{AZ}^{+}\right)}\right] /\left[\frac{\varepsilon\left({ }^{3} \mathrm{AZ} Z^{*}\right)}{\varepsilon\left(\mathrm{AZ}^{+}\right)}\right]
$$

which is $5.7 \%$ using the above parameters.

\section{References for SI:}

(1) Hou, J.; Inganäs, O.; Friend, R. H.; Gao, F. Organic solar cells based on non-fullerene acceptors. Nat. Mater. 2018, 17, 119.

(2) Jen, M.; Lee, S.; Jeon, K.; Hussain, S.; Pang, Y. Ultrafast Intramolecular Proton Transfer of Alizarin Investigated by Femtosecond Stimulated Raman Spectroscopy. J. Phys. Chem. B 2017, 121, 4129-4136.

(3) Amat, A.; Miliani, C.; Romani, A.; Fantacci, S. DFT/TDDFT investigation on the UV-vis absorption and fluorescence properties of alizarin dye. Phys. Chem. Chem. Phys. 2015, 17, 6374-6382.

(4) Ingole, P. P.; Markad, G. B.; Saraf, D.; Tatikondewar, L.; Nene, O.; Kshirsagar, A.; Haram, S. K. Band Gap Bowing at Nanoscale: Investigation of CdSxSe1-x Alloy Quantum Dots through Cyclic Voltammetry and Density Functional Theory. J. Phys. Chem. C 2013, 117, 7376-7383.

(5) Brus, L. E. A simple model for the ionization potential, electron affinity, and aqueous redox potentials of small semiconductor crystallites. J. Chem. Phys. 1983, 79, $5566-5571$. 